\title{
A note on the paper 'Fixed point theorems for cyclic weak contractions in compact metric spaces'
}

\author{
Zoran Kadelburg ${ }^{1}$, Stojan Radenović $2,3^{*}$ and Jelena Vujaković ${ }^{4}$
}

\section{"Correspondence:}

stojan.radenovic@tdt.edu.vn

${ }^{2}$ Nonlinear Analysis Research Group,

Ton Duc Thang University, Ho Chi

Minh City, Vietnam

${ }^{3}$ Faculty of Mathematics and

Statistics, Ton Duc Thang University, Ho Chi Minh City, Vietnam

Full list of author information is available at the end of the article

\begin{abstract}
We show that the result on cyclic weak contractions of Harjani et al. (J. Nonlinear Sci. Appl. 6:279-284, 2013) holds without the assumption of compactness of the underlying space, and also without the assumption of continuity of the given mapping.
\end{abstract}

MSC: $47 \mathrm{H} 10 ; 54 \mathrm{H} 25$

Keywords: compact metric space; cyclic contraction; weak contraction; well posed problem

\section{Introduction}

In 2003, Kirk et al. introduced in [2] an interesting concept of cyclic contractions in metric spaces, and obtained the corresponding generalizations of Banach's, as well as some other fixed point results. In subsequent articles, several authors obtained various fixed point theorems, adapting some other known results to their cyclic variants (see, e.g., [1, 3-9]).

The first results for cyclic contractions in compact metric spaces were obtained already in [2]. Recently, Harjani et al. [1] obtained a cyclic fixed point result in compact spaces for weak contractions (in the sense of [10,11]), thus modifying a theorem of Karapinar [4] and Karapinar and Sadarangani [5].

In this note, we are going to improve the result from [1] showing that it still holds without the assumption of compactness of the underlying space, and also without the assumption of continuity of the given mapping. An application to well posedness of the corresponding problem is also obtained.

\section{Preliminaries}

Let $X$ be a nonempty set, and $A_{1}, A_{2}, \ldots, A_{p}$ be its nonempty subsets. Recall that $Y=$ $\bigcup_{i=1}^{p} A_{i}$ is said to be a cyclic representation of $Y$ with respect to a mapping $T: Y \rightarrow Y$ if

$$
T\left(A_{1}\right) \subset A_{2}, \quad \ldots, \quad T\left(A_{p-1}\right) \subset A_{p}, \quad T\left(A_{p}\right) \subset A_{1} .
$$

Roughly speaking, there are two kinds of fixed point results for cyclic contractions - those assuming that $Y=X$, and those that do not use this assumption.

(c) 2016 Kadelburg et al. This article is distributed under the terms of the Creative Commons Attribution 4.0 International License (http://creativecommons.org/licenses/by/4.0/), which permits unrestricted use, distribution, and reproduction in any medium, provided you give appropriate credit to the original author(s) and the source, provide a link to the Creative Commons license, and indicate if changes were made. 
In $[4,5]$, the following class of functions was used:

$$
\begin{aligned}
\mathcal{J}= & \{\phi:[0,+\infty) \rightarrow[0,+\infty): \phi \text { is continuous, nondecreasing, } \phi(0)=0 \text { and } \phi(t)>0 \\
& \text { for } t \in(0,+\infty)\} .
\end{aligned}
$$

The main result in $[4,5]$ is the following.

Theorem 1 ([4], Theorem 6, [5], Theorem 2) Let $(X, d)$ be a complete metric space, and let $Y=\bigcup_{i=1}^{p} A_{i}$ be a cyclic representation of $Y \subseteq X$ with respect to a mapping $T: Y \rightarrow Y$, where the sets $A_{i}$ are closed. If, for some $\phi \in \mathcal{J}$,

$$
d(T x, T y) \leq d(x, y)-\phi(d(x, y))
$$

for any $x \in A_{i}$ and $y \in A_{i+1}, i=1,2, \ldots, p$, where $A_{p+1}=A_{1}$, then $T$ has a unique fixed point $z \in \bigcap_{i=1}^{p} A_{i}$.

We will use the following auxiliary result.

Lemma 1 ([9], Lemma 2.1) Let $(X, d)$ be a metric space, $T: X \rightarrow X$ be a mapping and let $X=\bigcup_{i=1}^{p} A_{i}$ be a cyclic representation of $X$ w.r.t. $T$. Assume that

$$
\lim _{n \rightarrow \infty} d\left(x_{n}, x_{n+1}\right)=0
$$

where $x_{n+1}=T x_{n}, x_{1} \in A_{1}, n \in \mathbb{N}$. If $\left\{x_{n}\right\}$ is not a Cauchy sequence, then there exist an $\varepsilon>0$ and two sequences $\{n(k)\}$ and $\{m(k)\}$ of positive integers such that $n(k)>m(k)>k$ and the following sequences tend to $\varepsilon^{+}$as $k \rightarrow \infty$ :

$$
\begin{array}{ll}
d\left(x_{m(k)-j(k)}, x_{n(k)}\right), & d\left(x_{m(k)-j(k)+1}, x_{n(k)}\right), \\
d\left(x_{m(k)-j(k)}, x_{n(k)+1}\right), & d\left(x_{m(k)-j(k)+1}, x_{n(k)+1}\right),
\end{array}
$$

where $j(k) \in\{1,2, \ldots, p\}$ is chosen so that $n(k)-(m(k)-j(k)) \equiv 1(\bmod p)$, for each $k \in \mathbb{N}$.

Remark 1 Using the previous lemma, similar to [9], one can prove that there is an equivalence between Theorem 1 and the corresponding fixed point result for non-cyclic weak contraction. In the case when $Y=X=\bigcup_{i=1}^{p} A_{i}$, no assumption on the closedness of sets $A_{i}$ is necessary, while in the case when $Y \neq X$, it is enough to assume that one of these sets is closed (similarly as in [3], Theorem 2.1). However, in this case the cyclic and non-cyclic versions of this assertion are not equivalent anymore.

In [1], the following class of functions (obviously wider than $\mathcal{J}$ ) was used in order to obtain a version of Theorem 1 in compact metric spaces (and assuming that $Y=X$ ):

$$
\begin{aligned}
\mathcal{F}=\{\phi:[0,+\infty) \rightarrow[0,+\infty): \phi \text { is nondecreasing, } \phi(0)=0 \text { and } \phi(t)>0 \\
\\
\text { for } t \in(0,+\infty)\} .
\end{aligned}
$$


Theorem 2 ([1], Theorem 2.1) Let $(X, d)$ be a compact metric space and $T: X \rightarrow X$ a continuous mapping. Suppose that $X=\bigcup_{i=1}^{p} A_{i}$ is a cyclic representation of $X$ with respect to $T$. If, for some $\phi \in \mathcal{F}$, relation (2.2) holds for any $x \in A_{i}$ and $y \in A_{i+1}, i=1,2, \ldots, p$ where $A_{p+1}=A_{1}$, then $T$ has a unique fixed point $z \in \bigcap_{i=1}^{p} A_{i}$.

Remark 2 Under the assumptions of Theorem 2, it can easily be seen that $X=\bigcup_{i=1}^{p} \overline{A_{i}}$ is also a cyclic representation of $X$ w.r.t. $T$. Moreover, all sets $\overline{A_{i}}, i \in\{1, \ldots, p\}$ are compact in $X$ and (2.2) implies that

$$
d(T x, T y)<d(x, y)
$$

holds for all $x \in A_{i}, y \in A_{i+1}$ with $x \neq y$. But then the result follows directly from [2], Theorem 2.1. Hence, Theorem 2 is not a new result.

Note also that [1], Remark 2.2, is not correct. Namely, Theorem 2 is not a generalization of Theorem 1, since it uses the assumption $Y=X$, which is not present in Theorem 1.

The following result is a version of [4], Theorem 7 , when $(X, d)$ is a compact metric space.

Theorem 3 ([1], Theorem 2.3) Under the assumptions of Theorem 2, the fixed point problem for $T$ is well posed, that is, if there exists a sequence $\left\{y_{n}\right\}$ in $X$ with $d\left(y_{n}, T y_{n}\right) \rightarrow 0$ as $n \rightarrow \infty$, then $y_{n} \rightarrow z$ as $n \rightarrow \infty$, where $z$ is the unique fixed point of $T$.

\section{Results}

Our main result is the following improvement of Theorem 2, that is, the main result from [1]. Note that compactness of the space is not assumed, nor is the continuity of $T$. Also, the subsets $A_{i}$ of $X$ need not be closed. Moreover, the proof is much shorter than the proof of the relevant theorem in [1].

Theorem 4 Let $(X, d)$ be a complete metric space and $T: X \rightarrow X$ be a mapping. Suppose that $X=\bigcup_{i=1}^{p} A_{i}$ is a cyclic representation of $X$ with respect to $T$. If, for some $\phi \in \mathcal{F}$, relation (2.2) holds for any $x \in A_{i}$ and $y \in A_{i+1}, i=1,2, \ldots, p$, where $A_{p+1}=A_{1}$, then $T$ has a unique fixed point $z \in X$ and $z \in \bigcap_{i=1}^{p} A_{i}$. Moreover, each Picard sequence $x_{n}=T^{n} x_{0}, x_{0} \in X$ converges to $z$.

Proof Take arbitrary $x_{0} \in X$. It belongs to some of the subsets $A_{i}, i \in\{1, \ldots, p\}$, say $x_{0} \in A_{1}$. Then the Picard sequence $x_{n}=T x_{n-1}, n \in \mathbb{N}$, is divided into the following $p$ subsequences, each belonging to some $A_{i}, i \in\{1, \ldots, p\}$ :

$$
\left\{x_{n p}\right\} \subset A_{1}, \quad\left\{x_{n p+1}\right\} \subset A_{2}, \quad \ldots, \quad\left\{x_{n p+p-1}\right\} \subset A_{p} .
$$

Suppose that $x_{n+1} \neq x_{n}$, for each $n$ (otherwise there is nothing to prove). It easily follows that $x_{n} \neq x_{m}$ for $n \neq m$. Moreover, it can be proved in a standard way (e.g., as in the proof of [1], Theorem 2.1) that

$$
d\left(x_{n}, x_{n+1}\right) \downarrow 0 \quad \text { as } n \rightarrow \infty .
$$

We will prove that $\left\{x_{n}\right\}$ is a Cauchy sequence. 
Suppose that this is not true. Then, using Lemma 1 , we conclude that there exist an $\varepsilon>0$ and two sequences $\{n(k)\}$ and $\{m(k)\}$ of positive integers, with $n(k)>m(k)>k$, such that the sequences (2.3) tend to $\varepsilon^{+}$when $k \rightarrow \infty$. Applying (2.2) with $x=x_{m(k)-j(k)}, y=x_{n(k)}$, we get

$$
d\left(x_{m(k)-j(k)+1}, x_{n(k)+1}\right) \leq d\left(x_{m(k)-j(k)}, x_{n(k)}\right)-\phi\left(d\left(x_{m(k)-j(k)}, x_{n(k)}\right)\right),
$$

i.e.,

$$
0<\phi\left(d\left(x_{m(k)-j(k)}, x_{n(k)}\right)\right) \leq d\left(x_{m(k)-j(k)}, x_{n(k)}\right)-d\left(x_{m(k)-j(k)+1}, x_{n(k)+1}\right),
$$

since $x_{m(k)-j(k)} \neq x_{n(k)}$ (which follows from $n(k)>m(k)$ ). Passing to the limit as $k \rightarrow \infty$, we get

$$
\phi\left(d\left(x_{m(k)-j(k)}, x_{n(k)}\right)\right) \rightarrow 0, \quad \text { as } k \rightarrow \infty .
$$

Since $\varepsilon \leq d\left(x_{m(k)-j(k)}, x_{n(k)}\right)$ for $k$ sufficiently large, we obtain $0<\phi(\varepsilon) \leq \phi\left(d\left(x_{m(k)-j(k)}, x_{n(k)}\right)\right)$, a contradiction. Hence, $\left\{x_{n}\right\}$ is a Cauchy sequence.

Since the space $X$ is complete, there is $z \in X=\bigcup_{i=1}^{p} A_{i}$ such that $x_{n} \rightarrow z$ as $n \rightarrow \infty$. This means that $z \in A_{m}$ for some $m \in\{1, \ldots, p\}$ and, hence, $T z \in A_{m+1}$. Consider the subsequence $\left\{x_{n p+m}\right\} \subset A_{m+1}$ of $\left\{x_{n}\right\}$. Applying (2.2), we get

$$
\begin{aligned}
d\left(x_{n p+m+1}, T z\right) & =d\left(T x_{n p+m}, T z\right) \\
& \leq d\left(x_{n p+m}, z\right)-\phi\left(d\left(x_{n p+m}, z\right)\right) \\
& \leq d\left(x_{n p+m}, z\right) \rightarrow 0, \quad \text { as } n \rightarrow \infty .
\end{aligned}
$$

It follows that $x_{n p+m+1} \rightarrow T z$ as $n \rightarrow \infty$ and, by the uniqueness of the limit, $T z=z$. By the cyclic property (2.1) of $T$, it follows that $z \in \bigcap_{i=1}^{p} A_{i}$.

The uniqueness of the fixed point follows from the contractive condition (2.2), since, again by (2.1), each fixed point of $T$ belongs to $\bigcap_{i=1}^{p} A_{i}$.

Taking $\phi(t)=(1-k) t, t \in[0,+\infty), k \in[0,1)$ in Theorem 4 , we obtain the following version of a Banach-type cyclic contraction result.

Corollary 1 Let $(X, d)$ be a complete metric space and $T: X \rightarrow X$ be a mapping. Suppose that $X=\bigcup_{i=1}^{p} A_{i}$ is a cyclic representation of $X$ with respect to $T$. If, for some $k \in[0,1)$, the inequality $d(T x, T y) \leq k d(x, y)$ holds for any $x \in A_{i}$ and $y \in A_{i+1}, i=1,2, \ldots, p$, where $A_{p+1}=A_{1}$, then $T$ has a unique fixed point $z \in X$ and $z \in \bigcap_{i=1}^{p} A_{i}$. Moreover, each Picard sequence $x_{n}=T^{n} x_{0}, x_{0} \in X$, converges to $z$.

Example 1 Let $X=\mathbb{R}$ be endowed with the standard metric. Take $A_{1}=(-\infty, 2), A_{2}=$ $(-2,+\infty)$, and define $T: X \rightarrow X$ and $\phi \in \mathcal{F}$ by

$$
T x=\left\{\begin{array}{ll}
-\frac{1}{2} x, & |x|<2, \\
-\frac{1}{3} x, & |x| \geq 2,
\end{array} \quad \phi(t)= \begin{cases}\frac{1}{2} t, & t \in[0,2), \\
\frac{1}{3} t, & t \in[2,+\infty) .\end{cases}\right.
$$


Then all the assumptions of Theorem 4 are fulfilled; let us check the condition (2.2). Suppose that $x \in A_{1}$ and $y \in A_{2}$ (the other case can be treated symmetrically). Consider the following possibilities:

(1) $x \in(-\infty,-2]$, (2) $x \in(-2,0)$, (3) $x \in[0,2)$, as well as: (a) $y \in(-2,0)$, (b) $y \in[0,2)$, (c) $y \in[2,+\infty)$.

Case 1(a). Tx $=-\frac{1}{3} x<-\frac{1}{2} x, T y=-\frac{1}{2} y$, and $d(T x, T y)<\frac{1}{2}(-x-(-y))=\frac{1}{2}(y-x)=\frac{1}{2} d(x, y)$.

Case 1(b). Tx $=-\frac{1}{3} x<-\frac{1}{2} x, T y=-\frac{1}{2} y, d(T x, T y)<\frac{1}{2}(-x-(-y))=\frac{1}{2}(y-x)=\frac{1}{2} d(x, y)$.

Case 1(c). Tx $=-\frac{1}{3} x, T y=-\frac{1}{3} y, d(T x, T y)=\frac{1}{3}(-x-(-y))=\frac{1}{3}(y-x)<\frac{1}{2} d(x, y)$.

Case 2(a). Tx $=-\frac{1}{2} x, T y=-\frac{1}{2} y, d(T x, T y)=\frac{1}{2}|x-y|=\frac{1}{2} d(x, y)$.

Case 2(b). $T x=-\frac{1}{2} x, T y=-\frac{1}{2} y, d(T x, T y)=\frac{1}{2}(x-y)=\frac{1}{2} d(x, y)$.

The cases 2(c), 3(a), 3(b), and 3(c) are symmetric to the cases 1(b), 2(b), 2(a), and 1(a), respectively. On the other hand, $\phi(t) \geq \frac{1}{2} t$ for all $t \geq 0$.

Hence, in all possible cases,

$$
d(T x, T y) \leq \frac{1}{2} d(x, y) \leq d(x, y)-\phi(d(x, y))
$$

and the contractive condition (2.2) of Theorem 4 is fulfilled. We conclude that $T$ has a unique fixed point (which is $z=0$ ). Since:

1. $X$ is not compact;

2. $T$ is not continuous;

3. $A_{i}, i \in\{1,2\}$, are not closed,

Theorems 1 and 2 cannot be used to reach this conclusion.

The following modification of Theorem 4 can be proved nearly in the same way.

Theorem 5 Let $X$ be a nonempty set, and $A_{1}, A_{2}, \ldots, A_{p}$ be its nonempty subsets, with at least one of them being closed. Let $Y=\bigcup_{i=1}^{p} A_{i}$ be a cyclic representation of $Y$ with respect to a mapping $T: Y \rightarrow Y$. If, for some $\phi \in \mathcal{F}$, relation (2.2) holds for any $x \in A_{i}$ and $y \in$ $A_{i+1}, i=1,2, \ldots, p$, where $A_{p+1}=A_{1}$, then $T$ has a unique fixed point $z \in Y$ and $z \in \bigcap_{i=1}^{p} A_{i}$. Moreover, each Picard sequence $x_{n}=T^{n} x_{0}, x_{0} \in Y$ converges to $z$.

Proof Suppose, without loss of generality, that the subset $A_{1}$ is closed (and hence complete) in $X$. The only difference in the proof, compared with Theorem 4 , is that, after proving that $\left\{x_{n}\right\}$ is a Cauchy sequence (in $Y$ ), we conclude that there is $z \in A_{1}(\subseteq Y$ ) such that $x_{n} \rightarrow z$, as $n \rightarrow \infty$. See also the proof of [3], Theorem 2.1.

As another consequence of Theorem 4, we obtain the following improvement of Theorem 3 .

Corollary 2 Under the assumptions of Theorem 4, the fixed point problem for $T$ is well posed, that is, if $\left\{y_{n}\right\}$ is a sequence in $X$ satisfying $d\left(y_{n}, T y_{n}\right) \rightarrow 0$ as $n \rightarrow \infty$, then $y_{n} \rightarrow z$ as $n \rightarrow \infty$, where $z$ is the unique fixed point of $T$ (whose existence is guaranteed by Theorem 4 ).

Proof Since, according to Theorem 4, it follows that the unique fixed point $z$ of $T$ belongs to $\bigcap_{i=1}^{p} A_{i}$, the rest of the proof is the same as in [1], p. 282. Namely, this proof uses neither compactness of $X$, nor continuity of $T$. 
Remark 3 As a kind of conclusion, we state once more that there are two kinds of cyclic fixed point results - those treating mappings $T: Y \rightarrow Y$, where $Y=\bigcup_{i=1}^{p} A_{i} \subset X$ (possibly with $Y \neq X$ ) and those where $Y=X$. In results of the first kind, it is enough to assume that one of the sets $A_{i}$ is closed, while in results of the second kind, no closedness assumption is needed. In both cases, if the considered mapping $T$ is continuous, all the results reduce to the case when all $A_{i}$ 's are closed.

\section{Competing interests}

The authors declare that they have no competing interests.

\section{Authors' contributions}

All authors contributed equally and significantly in writing this paper. All authors read and approved the final manuscript.

\section{Author details}

${ }^{1}$ Faculty of Mathematics, University of Belgrade, Beograd, Serbia. ${ }^{2}$ Nonlinear Analysis Research Group, Ton Duc Thang University, Ho Chi Minh City, Vietnam. ${ }^{3}$ Faculty of Mathematics and Statistics, Ton Duc Thang University, Ho Chi Minh City, Vietnam. ${ }^{4}$ Faculty of Sciences and Mathematics, University in Priština, Lole Ribara 29, Kosovska Mitrovica, 38220, Serbia.

\section{Acknowledgements}

The authors are indebted to the referees of this paper who helped us to improve the text. The first author is thankful to the Ministry of Education, Science and Technological Development of Serbia, Grant No. 174002.

Received: 27 January 2016 Accepted: 30 March 2016 Published online: 06 April 2016

\section{References}

1. Harjani, J, Lopez, B, Sadarangani, K: Fixed point theorems for cyclic weak contractions in compact metric spaces. J. Nonlinear Sci. Appl. 6, 279-284 (2013)

2. Kirk, WA, Srinavasan, PS, Veeramani, P: Fixed points for mapping satisfying cyclical contractive conditions. Fixed Point Theory 4, 79-89 (2003)

3. Jleli, M, Samet, B: An improvement result concerning fixed point theory for cyclic contractions (submitted)

4. Karapınar, E: Fixed point theory for cyclic weak $\phi$-contraction. Appl. Math. Lett. 24, 822-825 (2011)

5. Karapınar, E, Sadarangani, K: Corrigendum to 'Fixed point theory for cyclic weak $\phi$-contraction' [Appl. Math. Lett. 24 (6) (2011) 822-825]. Appl. Math. Lett. 25, 1582-1584 (2012)

6. Magdas, A: Fixed point theorems for Ćirić type generalized contractions defined on cyclic representations. J. Nonlinear Sci. Appl. 8, 1257-1264 (2015)

7. Petric, MA: Some results concerning cyclical contractive mappings. Gen. Math. 18(4), 213-226 (2010)

8. Pacurar, M, Rus, IA: Fixed point theory for cyclic $\varphi$-contractions. Nonlinear Anal. 72, 1181-1187 (2010)

9. Radenović, S: A note on fixed point theory for cyclic $\varphi$-contractions. Fixed Point Theory Appl. 2015, 189 (2015)

10. Alber, YI, Guerre-Delabriere, S: Principle of weakly contractive maps in Hilbert space. In: Golberg, I, Lyubich, Y (eds.) New Results in Operator Theory and Its Applications. Operator Theory: Advances and Applications, vol. 98, pp. 7-22. Birkhäuser, Basel (1997)

11. Rhoades, BE: Some theorems on weakly contractive maps. Nonlinear Anal. 47, 2683-2693 (2001)

\section{Submit your manuscript to a SpringerOpen ${ }^{\circ}$ journal and benefit from:}

- Convenient online submission

Rigorous peer review

- Immediate publication on acceptance

- Open access: articles freely available online

- High visibility within the field

- Retaining the copyright to your article 